\title{
O cenário da desinstitucionalização em Pernambuco: perfil sociodemográfico e clínico de usuários de hospital psiquiátrico
}

\author{
The scenario of deinstitutionalization in Pernambuco, Brazil: \\ sociodemographic and clinical profile of psychiatric hospital users
}

\section{El escenario de la desinstitucionalización en Pernambuco, Brazil: perfil sociodemográfico y clínico de usuarios de hospital psiquiátrico}

Anna Carolina de Sena e Vasconcelos ${ }^{1}$ Vera Lúcia Dutra Facundes ${ }^{2}$

\begin{abstract}
RESUMO: Objetivo: traçar o perfil sociodemográfico e clínico dos usuários de longa permanência de um hospital psiquiátrico em Pernambuco que se encontra em processo de fechamento. Método: pesquisa descritiva realizada com dados secundários de 49 pacientes, obtidos pela Secretaria Estadual de Saúde de Pernambuco (SES/PE). Os dados foram analisados por estatística descritiva e apresentados em termos de frequência absoluta e porcentagem. Resultados: a população foi caracterizada como predominantemente do sexo masculino $(95,9 \%)$, analfabeta $(34,7 \%)$, solteira (71,5\%), com rede social ou de suporte limitada à família (42,9\%), com escassas informações sobre tipo ou fonte de renda (30,6\%), com período de hospitalização em torno de 6 anos, sem saída do hospital após a internação (38,9\%). Os principais diagnósticos foram esquizofrenia $(49,4 \%)$ e retardo mental (36,5\%). Conclusão: esta pesquisa visou a contribuir com a construção de políticas e ações voltadas à desinstitucionalização. Sugere-se o acompanhamento de processos como esse até seu desfecho.
\end{abstract}

Descritores: Desinstitucionalização, Hospitais psiquiátricos, Saúde mental, Serviços de saúde mental.

\begin{abstract}
Objective: outline the sociodemographic and clinical profile of long-stay users of a psychiatric hospital in Pernambuco, Brazil, which is going through a process of closing down. Method: descriptive study carried out using secondary data from 49 patients, obtained from the Pernambuco State Department of Health (SES/PE). Data were analyzed by descriptive statistics and shown in terms of absolute frequency and percentage. Results: the population was characterized as predominantly male $(95.9 \%)$, illiterate $(34.7 \%)$, single $(71.5 \%)$, having a social or support network limited to the family $(42.9 \%)$, having little information about the type or source of income $(30.6 \%)$, with a period of hospitalization around 6 years, without leaving the hospital after admission (38.9\%). The main diagnoses were schizophrenia (49.4\%) and mental retardation

1 Terapeuta Ocupacional pela UFPE. Especialização em Saúde Mental pelo Programa de Residência Multiprofissional do Instituto de Medicina Integral Professor Fernando Figueira (IMIP). Especialização (em andamento) em Saúde do Idoso pelo Programa de Residência Multiprofissional do Instituto de Medicina Integral Professor Fernando Figueira (IMIP). Universidade Federal de Pernambuco (UFPE). Recife, Pernambuco, Brasil. E-mail: annasena90@gmail.com 2 Terapeuta Ocupacional, mestre em Saúde Coletiva e doutora em Neuropsiquiatria e Ciências do Comportamento pela UFPE. Universidade Federal de Pernambuco/Departamento de Terapia Ocupacional (UFPE). Recife, Pernambuco, Brasil. E-mail: verafacundes@yahoo.com.br
\end{abstract}


(36.5\%). Conclusion: this research aimed to contribute to build policies and actions whose target is deinstitutionalization. We suggest monitoring processes like this until their outcome.

Descriptors: Deinstitutionalization, Psychiatric Hospitals, Mental Health, Mental Health Services.

RESUMEN : Objetivo: delinear el perfil sociodemográfico y clínico de los usuarios de larga estadía de un hospital psiquiátrico de Pernambuco, Brasil, que está atravesando un proceso de cierre. Método: investigación descriptiva realizada con datos secundarios de 49 pacientes, obtenidos del Departamento de Salud del Estado de Pernambuco (SES/PE). Los datos se analizaron por estadística descriptiva y se muestran en términos de frecuencia absoluta y porcentaje. Resultados: la población se caracterizó por ser predominantemente masculina $(95,9 \%)$, analfabeta $(34,7 \%)$, soltera $(71,5 \%)$, con una red social o de apoyo limitada a la familia (42,9\%), con poca información sobre el tipo o fuente de ingresos $(30,6 \%)$, con un período de hospitalización de alrededor de 6 años, sin salir del hospital después de la admisión (38,9\%). Los principales diagnósticos fueron esquizofrenia $(49,4 \%)$ y retraso mental $(36,5 \%)$. Conclusión: esta investigación tuvo como objetivo contribuir a la construcción de políticas y acciones dirigidas a la desinstitucionalización. Sugerimos monitorear procesos como este hasta su desenlace.

Descriptores: Desinstitucionalización, Hospitales Psiquiátricos, Salud Mental, Servicios de Salud Mental.

\section{INTRODUÇÃO}

Os hospitais psiquiátricos, caracterizados pelas internações em tempo integral e de longa duração, alcançaram a segunda metade do século XX como alvo de denúncias por violação dos direitos humanos e baixa eficácia reabilitadora no tratamento de pessoas com transtornos mentais. Tais críticas a esse modelo de atenção originaram reformas no setor, oriundas dos Estados Unidos e da Europa, com dois segmentos distintos: um que defendia a psiquiatria reformada, implicando a reformulação interna dessas instituições para que se tornassem, de fato, terapêuticas, e outro que propunha estender a psiquiatra ao espaço público1.

O Movimento da Reforma Psiquiátrica Brasileira (MRPB) teve início em 1970, influenciado pelas ideias do movimento italiano, através da mobilização e denúncia da "indústria da loucura", na qual o país experimentava uma expansão significativa das internações psiquiátricas. Por exemplo, no período que se seguiu ao golpe militar de 1964, pode-se observar a consolidação da articulação entre internação asilar e privatização da assistência, com a crescente contratação de leitos nas clínicas e hospitais psiquiátricos conveniados remuneradas pelo setor público2.

Em busca de espaços que incorporassem diferentes atores - trabalhadores, usuários e familiares - o MRPB fomentou-se em diferentes eventos, principalmente com a $8^{\text {a }}$ Conferência Nacional de Saúde, em 1986 em Brasília-DF; a 1ª Conferência Nacional de Saúde Mental em 1987, no Rio de Janeiro e o no $2^{\circ}$ Encontro Nacional dos Trabalhadores em Saúde Mental, também em 1987, em Bauru. Este último configurou-se como o marco da articulação de diferentes movimentos sociais em torno da Reforma Psiquiátrica, onde foi pactuado o lema "por uma sociedade sem manicômios"3.

Entre os eventos internacionais que contribuíram para as mudanças na saúde mental brasileira, encontra-se a Conferência Regional para a Reestruturação da Assistência Psiquiátrica 
realizada em 1990, em Caracas, sob o patrocínio da Organização Pan-Americana de Saúde. Neste evento, com representação do Ministério da Saúde brasileiro, foi promulgado o documento final intitulado "Declaração de Caracas", no qual os países participantes comprometiam-se a desenvolver a reestruturação da assistência psiquiátrica, revisar criticamente o papel hegemônico e centralizador do hospital psiquiátrico na prestação de serviços, salvaguardar a dignidade pessoal e os direitos humanos e civis, em um modelo de serviço de saúde comunitário ${ }^{4}$.

A percepção de que o cuidado prestado nos hospitais psiquiátricos brasileiros eram inadequados fomentou ações legislativas em meados de 1980, como o projeto de Lei n 3657/1989, do deputado Paulo Delgado, precursor da Lei Ordinária $n^{\circ}$ 10216/2001 que dispõe sobre a proteção e os direitos das pessoas portadoras de transtornos mentais e redireciona o modelo assistencial em saúde mental. Na esfera estadual, o Rio Grande do Sul atuou de forma pioneira e apresentou um marco político significativo, com a aprovação da Lei Estadual n 9.716/1992 que prescreveu sobre a reforma psiquiátrica no Rio Grande do Sul, incitando outros estados brasileiros ${ }^{5}$.

Não obstante, em Pernambuco, a Assembléia Legislativa promulgou a Lei n¹1.064/1994 que dispunha sobre a substituição progressiva dos Hospitais Psiquiátricos pela rede de atenção integral à saúde mental, que naquele momento não contava com as estratégias de desinstitucionalização previstas na Rede de Atenção Psicossocial (RAPS) instituída pela Portaria n 3088/2011. Porém, mesmo as estratégias de desinstitucionalização propostas pela RAPS já foram subsidiadas por projetos criados em períodos anteriores como os Serviços Residenciais Terapêuticos pela Portaria $\mathrm{n}^{\circ} 106 / 2000$ e o Programa de Volta para Casa criado pela Lei $\mathrm{n}^{\circ} 10.708 / 2003$.

Frente a este cenário, constata-se que a desconstrução do hospital psiquiátrico como organização e como instituição tem sido efetivada por meio de uma luta política, teórica e prática que aponta para uma rede comunitária de serviços ${ }^{3}$. Ou seja, a desinstitucionalização surge como o deslocamento do centro da atenção da instituição para a comunidade, e em caráter de desconstrução de um modelo arcaico centrado na doença para tratar o sujeito em suas condições concretas de vida, que não em cativeiro ${ }^{6}$.

Contudo, para o fechamento adequado dos hospitais psiquiátricos torna-se imprescindível uma saída planejada e assistida dos usuários, assim como, a criação de outros recursos na comunidade que sirvam de suporte social, na reinserção, reabilitação e (re)constituição da cidadania ${ }^{3}$. Além disso, é necessário frisar a reconstrução da prática clínica para que não sejam reproduzidas as intervenções reducionistas dessas instituições, tais como as estratégias de normalização e disciplinamento. Mas sim, reinventar a essa prática clínica como construção de possibilidades e subjetividades, responsabilizando-se pelo sofrimento humano e outros paradigmas centrados no cuidado, na tomada de responsabilidade e na cidadania enquanto princípio ético ${ }^{6}$.

No Nordeste, a reforma da Assistência a Psicopatas de Pernambuco implementada por Ulysses Pernambucano, a partir de 1930 trouxe mudanças importantes no cenário da saúde mental da época que permanecem atuais sendo parte constituinte das diretrizes nacionais da atenção à 
saúde mental. O estudo realizado por Facundes, Bastos, Vasconcelos, Lima Filho ${ }^{7}$ apontou que entre os anos de 2006 a 2009 houve uma redução progressiva de 16\% dos leitos em hospitais psiquiátricos e a implantação concomitante, mas não suficiente, de uma rede de serviços extrahospitalares concentrados na região metropolitana.

Dados da Secretaria Estadual de Saúde de Pernambuco fazem referência ao funcionamento de oito hospitais psiquiátricos, sendo três públicos e cinco privados, que juntos totalizam 1.073 leitos, embora, nos últimos cinco anos, tenham sido fechados seis hospitais. Entre os serviços privados, quatro se encontram em processo de descredenciamento do Sistema Único de Saúde $(\mathrm{SUS})^{8}$.

Apesar da magnitude epidemiológica dos transtornos mentais que os tornam um dos principais pontos da agenda estratégica da Organização Mundial de Saúde, a produção de pesquisas desenvolvidas com o enfoque na população de moradores de hospitais psiquiátricos encontra-se limitada pela fragilidade de estrutura da rede de serviços de saúde mental do SUS, programas de formação na área ou pela ausência como prioridade de pesquisa construída por convocação da política pública ${ }^{9-10}$.

Por conseguinte, na conjuntura do MRPB torna-se indispensável, com o auxílio da ciência, conhecer a população de longa permanência dos hospitais psiquiátricos e traçar a melhor forma de desinstitucionalizar esses pacientes e o destino mais adequado após a alta hospitalar ${ }^{10}$. Logo, o presente estudo teve por objetivo traçar o perfil sóciodemográfico e clínico dos usuários de longa permanência em um dos hospitais psiquiátricos de Pernambuco em processo de descredenciamento.

\section{MÉTODO}

Trata-se de uma pesquisa do tipo descritiva por meio de dados secundários obtidos da Secretaria Estadual de Saúde de Pernambuco (SES/PE), acerca do processo de descredenciamento do Sistema Único de Saúde (SUS) de um hospital psiquiátrico conveniado, localizado na região metropolitana do Recife/PE. Os dados consistem nas informações registradas em instrumento do próprio órgão, utilizados após autorização por meio de declaração de anuência do órgão.

O instrumento analisado foi elaborado pela SES/PE em 2010 voltado para o fechamento de hospitais psiquiátricos no estado e consiste em um censo com 68 itens, distribuídos em 6 categorias (Figura 1). 
Figura 1. Descrição das categorias temáticas do instrumento da Secretaria Estadual de Saúde de Pernambuco

\begin{tabular}{|c|c|}
\hline Categoria & Descrição \\
\hline Identificação & $\begin{array}{l}\text { Nome completo, data de nascimento, sexo, nacionalidade, } \\
\text { naturalidade, filiação, endereço de referência, documentação } \\
\text { (registro geral e cadastro de pessoa física), religião e cor }\end{array}$ \\
\hline Dados sociodemográficos & $\begin{array}{l}\text { Escolaridade, atividade produtiva ou laboral, vínculo com } \\
\text { instituição previdenciária e moradia }\end{array}$ \\
\hline Situação jurídica & Curatela \\
\hline Vínculos sociais & $\begin{array}{l}\text { Estado civil, vínculo com familiares, amigos, vizinhos ou pessoas } \\
\text { de grupos de assistência, manutenção desses vínculos por meio de } \\
\text { visitas no período de internação, realização e condições de saída da } \\
\text { instituição no período do internamento }\end{array}$ \\
\hline $\begin{array}{l}\text { Percurso institucional e } \\
\text { dados clínicos-psiquiátricos }\end{array}$ & $\begin{array}{l}\text { Assistência à saúde antes da internação atual, data da primeira } \\
\text { internação psiquiátrica e da atual, duração da internação atual, } \\
\text { hipótese diagnóstica, tratamento e atendimento por equipe } \\
\text { multidisciplinar }\end{array}$ \\
\hline Processo de desinstitucionalização & $\begin{array}{l}\text { Avaliação do nível de dependência do usuário na realização das } \\
\text { atividades de vida diária, contexto institucional e indicações } \\
\text { terapêuticas para o projeto de alta hospitalar }\end{array}$ \\
\hline
\end{tabular}

FONTE: dados secundários obtidos da Secretaria Estadual de Saúde de Pernambuco (SES/PE)

A coleta de dados ocorreu no segundo semestre de 2015 após a finalização da etapa de preenchimento do instrumento pelos profissionais da SES/PE. Os dados obtidos do instrumento foram selecionados de quatro categorias e analisados por estatística descritiva, apresentados em termos de frequência absoluta e porcentagem, por meio da planilha eletrônica Microsoft Excel 2007 para Windows 7. Definiram-se as hipóteses diagnósticas de acordo com a $10^{\text {a }}$ Classificação Internacional de Doenças (CID-10) ${ }^{11}$.

A pesquisa foi desenvolvida de acordo com os procedimentos éticos preconizados pela Resolução 466/12 do Conselho Nacional de Saúde (CNS) e aprovada pelo do Comitê de Ética em Pesquisas com Seres Humanos do Instituto de Medicina Integral Professor Fernando Figueira IMIP (CAAE no 49887915.7.0000.5201).

\section{RESULTADOS}

A população pesquisada era constituída por 49 usuários de longa permanência, ou seja, com período de um ano ou mais de internação, de um hospital psiquiátrico na região metropolitana do Recife/PE.

A Tabela 1 apresenta o perfil sociodemográfico desses usuários. A maior parte formada pelo ISSN 1982-8829 Tempus, actas de saúde colet, Brasília, 11(3), 22-35, 2017 - Epub mar, 2018 
sexo masculino, sendo 95,9\% homens e 4,1\% mulheres. A média de idade foi 46,5 $( \pm 11,7)$ anos, informação ignorada para 8 usuários.Em relação a escolaridade, a maioria era analfabeta $(34,7 \%)$, sem registro quanto ao ensino superior. No momento da internação, 32,7\% exerciam alguma ocupação, sendo esta informação ignorada para 53\%. As ocupações listadas foram: agricultor, auxiliar de pedreiro, balconista, cabeleireiro, capinador, carroceiro, empregada doméstica, estofador, gari, pintor, servente, vendedor e vigilante. $\mathrm{O}$ vínculo com instituição previdenciária foi ignorado na maior parte e registrado somente para $30,6 \%$ da população. A aposentadoria foi o principal tipo de renda apresentada $(28,6 \%)$.

Tabela 1. Dados sociodemográficos de usuários de longa permanência de um hospital psiquiátrico de Pernambuco em processo de desinstitucionalização

\begin{tabular}{lcc}
\hline Sexo & Masculino & $\%$ \\
Feminino & 47 & 95,9 \\
Escolaridade & 2 & 4,1 \\
Analfabeto & & 34,7 \\
Alfabetizado & 17 & 2 \\
$1^{\circ}$ Grau incompleto & 1 & 20,4 \\
$1^{\circ}$ Grau completo & 10 & 4,1 \\
$2^{\circ}$ Grau incompleto & 2 & 6,1 \\
$2^{\circ}$ Grau completo & 3 & 4,1 \\
Ignorado & 2 & 28,6 \\
Ocupação à época da internação & 14 & 14,3 \\
Não possuía & & 32,7 \\
Possuía & 7 & 53 \\
Ignorado & 16 & 22,5 \\
Vínculo com instituição previdenciária & 26 & 30,6 \\
Não possui & & 46,9 \\
Possui & 11 & \\
Ignorado & 15 & 18,4 \\
Tipo de renda & 23 & 14,3 \\
Sem renda & & 28,6 \\
Benefício & & 38,7 \\
Outros (aposentadoria) & 9 & \\
Ignorado & 7 & 14 \\
\hline
\end{tabular}

As questões relacionadas aos vínculos sociais e aspectos jurídicos foram expostas na Tabela 2. O estado civil com maior representação foi o de solteiro (71,5\%). Posto que $42,9 \%$ dos usuários tenham a família como rede social ou de suporte, apenas $18,4 \%$ possuíam registro de curatela. As visitas dos familiares ocorreram para $44,9 \%$ dessa população, com frequência semanal em $20,4 \%$ dos casos.

Embora internados em regime fechado, 26,5\% dos usuários tinham um termo fornecido pelo responsável, anexado em prontuário, com a autorização e as devidas condições para sair da 
instituição. Entre as condições para saída 22,4\% necessitavam de acompanhante e 2\% poderia sair sem acompanhante. Não obstante, 38,9\% dos usuários nunca saíram do hospital após a internação.

Tabela 2. Vínculos sociais e aspectos jurídicos de usuários de longa permanência de um hospital psiquiátrico de Pernambuco em processo de desinstitucionalização

\begin{tabular}{|c|c|c|}
\hline & $\mathrm{N}$ & $\%$ \\
\hline \multicolumn{3}{|l|}{ Estado civil } \\
\hline Solteiro & 35 & 71,5 \\
\hline Casado / União estável & 1 & 2 \\
\hline Separado & 1 & 2 \\
\hline Ignorado & 12 & 24,5 \\
\hline \multicolumn{3}{|l|}{ Curatela } \\
\hline Sob curatela & 9 & 18,4 \\
\hline Sem curatela & 8 & 16,3 \\
\hline Ignorada & 32 & 65,3 \\
\hline \multicolumn{3}{|l|}{ Rede social ou de suporte } \\
\hline Não identificada & 13 & 26,5 \\
\hline Familiar & 21 & 42,9 \\
\hline Ignorada & 15 & 30,6 \\
\hline \multicolumn{3}{|l|}{ Visitação } \\
\hline Não recebia visitas & 12 & 24,5 \\
\hline Familiares & 22 & 44,9 \\
\hline Ignorada & 15 & 30,6 \\
\hline \multicolumn{3}{|c|}{ Frequência das visitas durante a internação } \\
\hline Não recebe visitas & 12 & 24,5 \\
\hline Semanal & 10 & 20,4 \\
\hline Quinzenal & 5 & 10,2 \\
\hline Mensal & 4 & 8,2 \\
\hline Eventual & 3 & 6,1 \\
\hline Ignorada & 15 & 30,6 \\
\hline \multicolumn{3}{|c|}{ Saída do usuário da instituição durante a internação } \\
\hline Não sai da instituição & 19 & 38,8 \\
\hline Semanal & 1 & 2 \\
\hline Quinzenal & 2 & 4,1 \\
\hline Eventual & 10 & 20,4 \\
\hline Ignorada & 17 & 34,7 \\
\hline \multicolumn{3}{|c|}{ Condições para saída do usuário da instituição durante a internação } \\
\hline Não sai da instituição & 19 & 38,9 \\
\hline Sai sem acompanhante & 1 & 2 \\
\hline Sai com acompanhante & 11 & 22,4 \\
\hline Ignorada & 18 & 36,7 \\
\hline
\end{tabular}

A Tabela 3 apresenta informações sobre o percurso institucional e dados clínicos. Observa-se que 53,1\% já recebia assistência por meio de hospital psiquiátrico, dando continuidade ao tratamento, ora por nova internação, ora por transferência de outra instituição. Não houve registro de assistência nos serviços substitutivos da RAPS, embora o município tivesse em sua 
rede dois Centros de Atenção Psicossocial e uma equipe de Consultório de Rua. Os dados apontam que $43 \%$ dos usuários foram internados no intervalo de 4 a 6 anos e 16,3\%, por 10 anos ou mais. Este período foi ignorado para $6,1 \%$ dos usuários, para os quais não constava registro da data de internação atual.

No que se refere as hipóteses diagnósticas, 49,4\% estavam relacionadas à esquizofrenia e $36,5 \%$ a retardo metal. Dos casos de transtorno mental devido ao uso de substância psicoativa, dois foram por uso de álcool e um por uso de múltiplas drogas, com períodos de internação de 14, 9 e 2 anos, respectivamente. Os registros apontam que $89,8 \%$ dos usuários realizavam tratamento com múltiplos medicamentos, enquanto para $10,2 \%$ esta informação foi ignorada.

Tabela 3. Percurso institucional e dados clínicos-psiquiátricos

\begin{tabular}{lcc}
\hline Assistência à saúde antes da internação atual & $\mathrm{N}$ & $\%$ \\
Não recebia assistência antes da internação & 1 & 2 \\
Hospital psiquiátrico & 26 & 53,1 \\
Outros & 4 & 8,2 \\
Ignorada & 18 & 36,7 \\
Duração da internação atual & & \\
1 a 3 anos & 8 & 16,3 \\
4 a 6 anos & 21 & 43 \\
7 a 9 anos & 9 & 18,3 \\
10 anos ou mais & 8 & 16,3 \\
Ignorada & 3 & 6,1 \\
Hipótese diagnóstica & & \\
Esquizofrenia & 24 & 49,4 \\
Retardo mental & 5 & 10,2 \\
Retardo mental e outros transtornos mentais & 13 & 26,3 \\
Transtorno mental devido ao uso de substância psicoativa & 3 & 6 \\
Outros transtornos & 2 & 4 \\
Ignorada & 2 & 4,1 \\
Informações sobre tratamento medicamentoso & & \\
Múltiplos medicamentos & 44 & 89,8 \\
Ignorada & 5 & 10,2 \\
\hline
\end{tabular}

Os atendimentos pela equipe multiprofissional não alcançaram a totalidade da população, como apontado na Tabela 4. A enfermagem e o serviço social assistiram o maior número de usuários $(85,7 \%)$, seguidas pela psicologia $(81,6 \%)$ e terapia ocupacional $(79,6 \%)$. Das especialidades médicas, psiquiatria e clínica médica atenderam $83,7 \%$ e $53,1 \%$ dos usuários, respectivamente. 
Tabela 4. Atendimento por categorial profissional aos usuários de longa permanência de um hospital psiquiátrico de Pernambuco em processo de desinstitucionalização

\begin{tabular}{lcc}
\hline & $\mathrm{N}$ & 85,7 \\
Enfermagem & 42 & 85,7 \\
Serviço social & 42 & 83,7 \\
Psiquiatria & 41 & 81,6 \\
Psicologia & 40 & 79,6 \\
Terapia ocupacional & 39 & 53,1 \\
Clínica médica & 26 & 6,1 \\
Nutrição & 3 & 2 \\
Fisioterapia & 1 & 2 \\
Odontologia & 1 & 12,2 \\
Ignorado & 6 & 2 \\
\hline
\end{tabular}

\section{DISCUSSÃO}

Os registros sobre a história da psiquiatria no nordeste apontam que Recife e Olinda receberam por iniciativa do poder público e das Santas Casas, um dos primeiros estabelecimentos no país destinados exclusivamente à pacientes com transtornos psiquiátricos, o Hospício da Visitação de Santa Isabel, em 1864, substituído em 1883, pelo Hospício de Alienados, a Tamarineira ${ }^{12}$. Cerca de um século e meio depois, a permanência de instituições desse tipo com o financiamento do poder público retorna a discussão com o descredenciamento do hospital psiquiátrico olindense do SUS.

A partir da análise sobre os resultados dos usuários do hospital psiquiátrico conveniado, localizado na região metropolitana do Recife/PE, a população pode ser caracterizada como de maioria do sexo masculino, analfabeta, solteira, com rede social ou de suporte limitada a família, com escassez de informações sobre tipo ou fonte de renda, com período de hospitalização em torno de 6 anos, na qual a maioria não havia saído do hospital após a internação, e diagnósticos relacionados à esquizofrenia e retardo mental. Estudos recentes em outros estados das regiões nordeste e sudeste corroboram com os dados encontrados em Pernambuco, nos quais a maioria da população institucionalizada em hospitais psiquiátricos brasileiros é composta por homens, numa faixa etária economicamente ativa, solteira, com vínculos sociais fragilizados, que vivem permanentemente no hospital ou não tinham licença para sair dele. Todavia, não foram encontrados estudos sobre o atendimento por equipe multiprofissional, limitando-se a relação de recursos humanos ligados ao campo da medicina ou enfermagem ${ }^{10,13-14}$.

Quanto aos aspectos socioeconômicos da população estudada, destacam-se como os principais tipos de renda a aposentadoria e o Benefício de Prestação Continuada (BPC), instituídas pelas leis $n^{\circ} 8.213 / 1991$ e $n^{\circ} 8.742 / 1993$, e alteradas pelas de $n^{\circ} 13.457 / 2017$ e $n^{\circ} 12.470 / 2011$, respectivamente. Observadas as condições do Instituto Nacional do Seguro Social (INSS) para concessão da aposentadoria por invalidez ${ }^{15-16}$, infere-se que estes usuários estiveram formalmente empregados por um período mínimo de um ano, e para o $\mathrm{BPC}^{17-18}$, que tenha sido comprovada a 
condição de pessoa com deficiência, assim como a impossibilidade de prover a própria manutenção nem de tê-la provida por sua família. Entretanto, as condições da presente pesquisa não permitiram explanar os motivos relacionados à permanência e saída do mercado de trabalho, assim como, a inclusão na Política de Seguridade Social não contributiva.

A importância do trabalho nesse contexto se dá na influência positiva sobre a saúde mental e funcionamento global do indivíduo, ressaltados pela melhora da autoestima, do funcionamento psicológico e controle dos sintomas quando comparados a indivíduos sem trabalho ou subempregados ${ }^{19}$. Para Andrade, Burali, Vida, Fransozio, Santos (2013), a questão do trabalho constitui um dos eixos do MRPB junto à retaguarda assistencial por meio dos serviços substitutivos, de moradia através do Sistema de Residências Terapêuticas e de reparação econômica através do programa De Volta para Casa, estabelecidos pela Portaria/GM n ${ }^{0} 106 / 2000$ e pela Lei Federal $10.708 / 2003$.

Além disso, eventos como a I Oficina Nacional de Experiências de Geração de Renda e Trabalho de Usuários de Serviços de Saúde Mental, realizada em 2004, pode ser tomada como marco histórico da constituição da Política Intersetorial de Saúde Mental e Economia Solidária, que possui como dispositivos institucionais a Rede Brasileira de Saúde Mental e Economia Solidária e o Cadastro de Iniciativas de Inclusão Social pelo Trabalho (CIST), do Ministério da Saúde. Tais dispositivos representariam uma das possibilidades de inclusão social dos usuários atendidos em serviços de saúde mental, resgatando a autonomia destes sujeitos do ponto de vista econômico ${ }^{20}$.

Os dados sobre os vínculos sociais dos usuários desta pesquisa nos mostram em sua maioria uma rede de suporte restrita a família, visitas com alguma periodicidade e a existência de condições de saída durante o período de internação. Observa-se que para os usuários que saem da instituição existe uma frequência e condição de acompanhamento, exceto para um usuário que poderia sair sem supervisão. Uma pesquisa realizada com familiares de ex-"moradores" de um hospital psiquiátrico cadastrados na Rede de Atenção Psicossocial de Maringá-PR, concluiu que

[...] a recorrência e longevidade da internação psiquiátrica dos "moradores" provocaram perda de papéis sociais e de laços afetivos, além de sinais de cronificação do transtorno mental, reforçando que o modelo manicomial acarreta agravos que poderiam ser evitados com o tratamento em consonância com os preceitos da atenção psicossocial (Frazatto, 2013:257).

Logo, se o papel do hospital especializado é a prestação de assistência à saúde, a longa permanência nos hospitais psiquiátricos não se justifica pela situação clínica, mas como lugar de moradia, por aspectos familiares, sociais, culturais, econômicos e políticos ${ }^{21}$.

O percurso institucional analisado reforça o que fora dito anteriormente sobre o tipo de assistência prestada pelo hospital psiquiátrico. Os principais diagnósticos encontrados neste estudo - esquizofrenia e retardo metal - possuem sintomas característicos que podem trazer inúmeras limitações relacionadas à linguagem e comunicação, à independência na realização das atividades básicas de vida diária, assim como, para atividades escolares, de trabalho ou lazer, e 
por consequência alcançar as expectativas da população na qual o indivíduo está inserido. Entre os prejuízos da internalização do estigma está o processo de transformação de identidade, em que o indivíduo passa a adotar uma visão estigmatizada sobre si mesmo, impedindo a formação de relações sociais benéficas ${ }^{22}$.

Ou seja, se a proposta de assistência da instituição não for de reabilitar para reinserir esse indivíduo, os problemas que o levaram à internação manter-se-ão como impeditivo para retornar à família e à comunidade. Por conseguinte, a importância e o compromisso dos equipamentos sociais no cuidado trazem representatividade aos princípios de promoção da saúde, prevenção de agravos e reabilitação psicossocial do SUS, uma vez que suas ações favorecem a construção social de espaços de vida e de expressão da diversidade ${ }^{23}$. Este fato pode ser observado no item sobre a assistência à saúde antes da internação atual, onde mais da metade dos usuários deram continuidade ao tratamento por nova internação ou por transferência de outro hospital psiquiátrico, sem registro de assistência em serviços substitutivos.

No Rio Grande do Norte ${ }^{24}$ foi realizada uma pesquisa no hospital psiquiátrico de referência, na qual foram identificadas como causas das dificuldades na assistência em saúde mental: o funcionamento inadequado dos serviços extra-hospitalares, dificuldade de acesso dos usuários aos serviços substitutivos, a problemática da dependência química no contexto da atenção psicossocial, dificuldade de usuários, familiares cuidadores e profissionais em aderir aos dispositivos da rede de saúde mental, desconhecimento de parcela dos profissionais do hospital psiquiátrico sobre a rede de atenção à saúde mental. Além do exposto, ressalta-se a influência dos estigmas sociais no acesso aos serviços de saúde nos diferentes setores, tais como na atenção básica ${ }^{25}$. No presente estudo, não foi possível identificar quais fatores influenciaram na escolha do serviço/local de assistência.

Em um contexto diferente no que concerne a assistência em saúde mental, mas não distante dos objetivos almejados no Brasil, um estudo europeu desenvolveu um instrumento específico e analisou a qualidade dos cuidados psiquiátricos e sociais para adultos com problemas de saúde mental a longo prazo. Nesta pesquisa internacional, constatou-se que a qualidade do atendimento foi maior em unidades menores, de sexo misto, com tempo de permanência máximo definido e onde nem todos os pacientes estivessem gravemente incapacitados ${ }^{26}$.

Ainda sobre a assistência em saúde mental, uma das principais queixas apresentadas nos serviços substitutivos é o déficit de psiquiatras ${ }^{24}$, porém mesmo em um hospital nesta área de especialidade, observou-se que não havia registro de atendimento médico para todos os usuários, assim como, para outras categorias profissionais. Isto é, a integralidade do atendimento perpassa os tipos de serviços disponibilizados em saúde mental. Entender que outros serviços podem assistir de maneira holística o indivíduo, visando à reabilitação psicossocial, mediante princípios como respeito e recontextualização das suas diferenças, preservação de sua identidade e cidadania por meio de uma participação ativa no tratamento ${ }^{27}$. No entanto, observa-se que

[...] apenas uma minoria da população consegue acesso ao cuidado de que necessita, e as instituições hospitalares que prestam esses cuidados têm se caracterizado, em sua maioria, 
pela violação dos direitos humanos dessa população e pela baixa eficácia reabilitadora (Lima, 2011: 2418)

A análise de estudos a cerca da desinstitucionalização em países desenvolvidos apontou que, por meio de um processo de reabilitação especializado ou intensivo, é possível otimizar o funcionamento social, estabilizar ou melhorar os sintomas psiquiátricos e, por conseguinte, a qualidade de vida destes usuários e sua relação com o ambiente ${ }^{28}$. De maneira geral, é necessário identificar e atuar sobre as diferentes áreas que possibilitem a recuperação dos usuários de saúde mental, neste caso, os de longa permanência ${ }^{29}$.

\section{CONCLUSÃO}

Após 152 anos da construção do primeiro hospital psiquiátrico em Pernambuco, as barreiras para a modificação da assistência aos usuários com transtorno mental não desapareceriam com uma lei promulgada há somente 15 anos. A longa permanência no hospital psiquiátrico permanece não se justificando pela situação clínica, mas sim como lugar de moradia. Muitos aspectos sociais, políticos e culturais estão envolvidos com essa realidade e precisam ser modificados para atender a uma população marginalizada pela sociedade e negligenciada pelos equipamentos de saúde e da assistência social.

Como apontado em outros estudos, embora haja a substituição progressiva dos leitos em hospitais psiquiátricos pela rede de atenção integralà saúde mental, a procura por serviços substitutivos necessita de uma reconfiguração no atendimento prestado, para que a desinstitucionalização desses usuários não se torne apenas uma desospitalização. A partir das informações deste estudo, supõe-se que a insuficiência da rede substitutiva mantém a busca pela assistência hospitalar.

Contudo, o uso de dados secundários foi uma fator limitante neste estudo, impedindo o aprofundamento e esclarecimento de algumas questões analisadas. Logo, sugere-se que sejam realizadas pesquisas direcionadas ao histórico ocupacional destes usuários, a articulação dos hospitais psiquiátricos com a rede substitutiva e ao acompanhamento de processos de desinstitucionalização até o desfecho.

\section{REFERÊNCIAS}

1. Batista MDG. Breve história da loucura, movimentos de contestação e reforma psiquiátrica na Itália,na França e no Brasil. Revista de Ciencias Sociais 2014; 40: 391-404.

2. Fonte, EM. Da institucionalização da loucura à reforma psiquiátrica: as sete vidas da agenda pública em saúde mental no Brasil. Estudos de Sociologia 2012; 1(18): 1-20.

3. Rameh-de-Albuquerque, RC, Lima WL, Costa AM, Nappo SA. Do descaso a um novo olhar: a construção da Política Nacional de Atenção Integral aos Usuários de Álcool e Outras Drogas como conquista da Reforma Psiquiátrica Brasileira. O caso de Recife (PE). Psicologia em Pesquisa 2017; 11(1); 84-96.

4. Lima FA. Saúde Mental à Luz da Psicologia Corporal. Revista Latino-Americana de Psicologia Corporal 2016; 3(5): $8-22$.

5. Goulart MSB. A política de saúde mental mineira: rumo à consolidação. Gerais: Revista Interinstitucional de ISSN 1982-8829 Tempus, actas de saúde colet, Brasília, 11(3), 22-35, 2017 - Epub mar, 2018 
Psicologia 2015; 8(2): 194-213.

6. Macedo JP, Abreu MM, Fontenele MG, Dimenstein, M. A regionalização da saúde mental e os novos desafios da Reforma Psiquiátrica brasileira. Saúde Soc 2017; 26(1): 155-170.

7. Facundes VLD, Bastos O, Vasconcelos MGL, Lima Filho IA. Atenção à Saúde Mental em Pernambuco: Perspectiva Histórica e Atual. Neurobiologia 2010; 73(1):183-197.

8. Secretaria Estadual de Saúde. Gerência de Atenção à Saúde Mental. Plano Estadual de Desinstitucionalização. Recife (PE); 2013.

9. Delgado PG. Limites para a inovação e pesquisa na reforma psiquiátrica. Physis 2015; 25(1):13-18.

10. Melo MCA, Albuquerque SGC, Luz JHS, Quental PTLF, Sampaio AM, Lima AB. Perfil clínico e psicossocial dos moradores em hospitais psiquiátricos no estado do Ceará, Brasil. Ciênc. saúde colet. 2015; 20(2): 343-352.

11. Organização Mundial da Saúde (OMS). CID-10: Classificação internacional de doenças e problemas relacionados a saúde. 10 a ed. São Paulo: EDUSP; 2017.

12. Santos, NMW. Loucura e sanidade psíquica: duas faces do desenvolvimento humano - alguns aspectos historiográficos (Brasil, 1808-2008). Revista Saúde e Desenvolvimento Humano 2013; 1(1): 61-72.

13. Pereira MO, Jericó MC, Perroca MG, Mukai HA. Long term psychiatric institution: profile of patients and human resource indicators. SMAD, Rev. Eletrônica Saúde Mental Álcool Drog. 2013;9(1):33-40.

14. Santos ARG, Lima CA, Santos ES, Bastos JF, Silva LG, Silveira HF, Ribeiro Junior HL. Perfil clínico dos pacientes com transtornos mentais internados em um hospital de custódia e tratamento - Bahia - Brasil. Rev. Ciênc. Méd. Biol. 2015; 14(2):190-197.

15. Brasil. Lei n. ${ }^{\circ} 8.213$ de 24 de julho de 1991. Dispõe sobre os Planos de Benefícios da Previdência Social e dá outras providências. Diário Oficial da União 25 jul 1991.

16. Brasil. Lei $\mathrm{n}^{\mathrm{o}} 13.457$ de 26 de junho de 2017 . Altera as Leis $\mathrm{n}$ os 8.213 , de 24 de julho de 1991 , que dispõe sobre os Planos de Benefícios da Previdência Social, e 11.907, de 2 de fevereiro de 2009, que dispõe sobre a reestruturação da composição remuneratória da Carreira de Perito Médico Previdenciário e da Carreira de Supervisor Médico-Pericial; e institui o Bônus Especial de Desempenho Institucional por Perícia Médica em Benefícios por Incapacidade. Diário Oficial da União 27 jun 2017.

17. Brasil. Lei n. ${ }^{\circ} 8.742$ de 7 de dezembro de 1993. Dispõe sobre a organização da Assistência Social e dá outras providências. Diário Oficial da União 8 dez 1993.

18. Brasil. Lei n 12.470 de 31 de agosto de 2011. Altera os arts. 21 e 24 da Lei no 8.212, de 24 de julho de 1991, que dispõe sobre o Plano de Custeio da Previdência Social, para estabelecer alíquota diferenciada de contribuição para o microempreendedor individual e do segurado facultativo sem renda própria que se dedique exclusivamente ao trabalho doméstico no âmbito de sua residência, desde que pertencente a família de baixa renda; altera os arts. 16, 72 e 77 da Lei no 8.213, de 24 de julho de 1991, que dispõe sobre o Plano de Benefícios da Previdência Social, para incluir o filho ou o irmão que tenha deficiência intelectual ou mental como dependente e determinar o pagamento do salário-maternidade devido à empregada do microempreendedor individual diretamente pela Previdência Social; altera os arts. 20 e 21 e acrescenta o art. 21-A à Lei no 8.742, de 7 de dezembro de 1993 - Lei Orgânica de Assistência Social, para alterar regras do benefício de prestação continuada da pessoa com deficiência; e acrescenta os $\S \S 4^{\circ}$ e e 5ao art. 968 da Lei no 10.406 , de 10 de janeiro de 2002 - Código Civil, para estabelecer trâmite especial e simplificado para o processo de abertura, registro, alteração e baixa do microempreendedor individual. Diário Oficial da União 31 ago 2011.

19. Assunção AA, Lima EP, Guimarães MDC. Transtornos mentais e inserção no mercado de trabalho no Brasil: um estudo multicêntrico nacional. Cad. Saúde Pública [Internet]. 2017[cited 2017 Dec 10]; 33(3):e00166815. Available from: http://www.scielo.br/pdf/csp/v33n3/1678-4464-csp-33-03-e00166815.pdf

20. Andrade MC, Burali MAM, Vida A, Fransozio MBB, Santos RZ. Loucura e Trabalho no Encontro entre Saúde

ISSN 1982-8829 Tempus, actas de saúde colet, Brasília, 11(3), 22-35, 2017 - Epub mar, 2018 
Mental e Economia Solidária. Psicologia: ciência e profissão, 2013, 33 (1), 174-191.

21. Frazatto CF, Boarini ML. O “morar" em hospital psiquiátrico: histórias contadas por familiares de ex-"moradores". Psicologia em Estudo, 2013; 18(2):257-267.

22. Ferreira GCL, Silveira OS, Noto AR, Ronzani TM. Implicações da relação entre estigma internalizado e suporte social para a saúde: Uma revisão sistemática da literatura. Estudos de Psicologia 2014; 19(1): 77-86.

23. Moura ACMD, Moura MLB, Facundes VLD, Lima Filho IA, Maranhão LCA, Borges MJL. A relação entre sujeitos com transtorno mental e equipamentos sociais. Cad. Ter. Ocup. UFSCar 2014; 22(2): 263-270.

24. Ramos DKR, Guimarães J, Mesquista SKC. Dificuldades da rede de saúde mental e as reinternações psiquiátricas: problematizando possíveis relações. Cogitare Enferm. 2014; 19(3):553-60.

25. Souza MLPD. Registro de distúrbios mentais no Sistema de Informação da Atenção Básica do Brasil, 2014. Epidemiologia e Serviços de Saúde, 2016;25(2):405-410.

26. Killaspy H et al. Quality of care and its determinants in longer term mental health facilities across Europe; a crosssectional analysis. BMC Psychiatry, 2016; 16(31):1-9.

27. Lima VBO, Branco Neto JRC. Reforma psiquiátrica e políticas públicas de saúde mental no Brasil: resgate da cidadania das pessoas portadoras de transtornos mentais. Direito \& Política, 2011; 1(1):121-31.

28. Kunitoh N. From hospital to the community: The influence of deinstitutionalization on discharged long-stay psychiatric patients. Psychiatry and Clinical Neurosciences, 2013; 67: 384-396.

29. Turton P, Wright C, White S, Killaspy H, DEMoBinc Group. Promoting Recovery in Long-Term Institutional Mental Health Care: An International Delphi Study. Psychiatric Services, 2010; 61(3):293-299.

Artigo apresentado em 30/10/2017

Artigo aprovado em 15/01/2018

Artigo publicado no sistema em 16/04/2018

ISSN 1982-8829 Tempus, actas de saúde colet, Brasília, 11(3), 22-35, 2017 - Epub mar, 2018 